\title{
Proyecto integral para agricultores de fresa y mora: Caso Tisaleo
}

\author{
Integral project for strawberry and blackberry farmers: Case Tisaleo
}

\author{
PAULINA SÁNCHEZa , FERNANDO SAÁb , JORGE ÁLVAREZ \\ aDiseño Gráfico, ${ }^{b}$ Carrera de Ingeniería Industrial, ' Centro de Transferencia Tecnológica \\ Universidad Tecnológica Indoamérica, Bolívar 20-35 y Guayaquil, Ambato-Ecuador \\ \{paulinasanchez, fernandosaa, jorgealvarez\}@uti.edu.ec
}

\section{RESUMEN}

El presente manuscrito pretende evidenciar los desafíos e intervenciones realizadas mediante el impulso de un proyecto de vinculación con la sociedad desarrollado por Universidad Tecnológica Indoamérica con la colaboración del Gobierno Autónomo Descentralizado de Tisaleo, estudiantes de las carreras de Ingeniería Industrial y Diseño Gráfico, y la asociación de agricultores de fresa y mora como beneficiarios directos. Inicialmente se ejecutó un diagnóstico situacional en el año 2017 con el respaldo de la información sobre los 6 representantes de la asociación más un representante del Municipio, para comprender el estado actual de la asociación de productores de fresa y mora de Tisaleo mediante entrevista no estructurada con dirigentes y agricultores, posteriormente se realizó un estudio de mercado y producción de fresa y mora para analizar la viabilidad de procesamiento de extracto de pulpa de los frutos, comercialización y difusión del producto en la feria, con estos datos se programa realizar proyecciones de maquinaria, capacitación de salud industrial y plan de campaña de difusión para dar a conocer el producto, su feria y mejorar los procesos de producción y contribuir directamente con la asociación de productores de fresa y mora del cantón Tisaleo.

Finalmente se plantea como propuesta optimizar los ingresos de los productores mediante el uso adecuado de las técnicas y las tecnologías para la comercialización de los productos así como la salud de los productores.

Palabras clave:

Agricultores, comercialización, producción, promoción, tecnologías. 


\section{ABSTRACT}

The current manuscript aims to highlight the challenges and interventions made by the impulse of a linkage project with the society developed at the University of Technology Indoamérica with the collaboration of the Decentralized Autonomous Government of Tisaleo, students of Engineering, Industrial and Graphic Design, and the association of farmers of strawberries and blackberries as direct beneficiaries. Initially they ran a diagnostic situation in the year 2017, with the information of 6 coworkers and 1 from the Municipality, to understand the current state of the association of producers of strawberries and blackberries of Tisaleo, through an interview is not structured with leaders and farmers, subsequently performed a market study and production of strawberries and blackberries to analyze the feasibility of processing of pulp extract of the fruits, commercialization and diffusion of the product in the fair, with these data it program to conduct projections of machinery, training of industrial health and plan outreach campaign to publicize the product, its fair and improve the production processes and directly contribute with the association of producers of strawberries and blackberries in the canton Tisaleo.

Finally, it raises as a proposal to optimise the income of the producers through appropriate use of techniques and technologies for the commercialization of the products as well as the health of the producers.

\section{Key words:}

Farmers, marketing, production, promotion, technology.

\section{INTRODUCCIÓN}

La agricultura ha sido practicada desde los inicios de la humanidad. Se han realizado modificaciones en los espacios agrícolas a través del tiempo; cambios producidos en función de la adaptación a los factores naturales como también en función de los sistemas económicos y políticos. "La agroindustria ya es un sector importante en muchas economías de los países basadas en la agricultura (ONUA, 2017). Ecuador se caracteriza por su tradicional desarrollo de los sectores agroexportadores que ha sido un factor determinante en la dinámica de la economía ecuatoriana.

La Sierra ha mantenido una línea productiva tradicional orientada a una demanda para el consumo interno y con un alto grado de autoconsumo que es lo que sucede en el caso de Tisaleo en la producción de fresa y mora que ha tenido auge hace 8 años atrás en la producción de fresa mientras que la ocupación del cultivo de mora se ha venido dando décadas atrás.

Las perspectivas de crecimiento de la demanda de mora siguen centrados en el consumo de los hogares y de la industria nacional, ya que, aunque existen nichos de mercado, las oportunidades de comercialización en fresco están limitadas por las restricciones fitosanitarias en el mercado nacional y la alta perecibilidad del producto que dificulta su transporte. La evolución de los precios mayoristas permite concluir que el mercado interno ha sido capaz de absorber la oferta creciente del producto en la última década.

A partir del 2009 se han proliferado los agricultores de fresa y mora dando paso a agrupaciones ubicadas en Quinchicoto, Santa Lucia

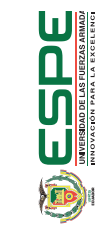


La Libertad, Santa Lucia Centro, centro Cantonal sumando 11 grupos que conforman la asociación de agricultores de fresa y mora quienes los días jueves a partir de las $14 \mathrm{~h} 00$ a $18 \mathrm{~h} 00$ comercializan su producto al por mayor y menor en el Mercado de Productores ubicado junto al Estadio Municipal del cantón Tisaleo en la Feria provincial; con apoyo del Gobierno Provincial de Tungurahua, GAD cantonal y la Mancomunidad del Frente Sur Occidental a través de la Estrategia Agropecuaria implementó para que la colectividad pueda adquirir directamente del productor al consumidor. (U. T. Indoamérica, 2018).

La Universidad Tecnológica Indoamérica con sus carreras y amparada en el convenio macro que mantiene con el Gobierno Provincial de Tungurahua y el GAD Municipal de Tisaleo ha venido trabajando en varios proyectos de vinculación; con los cuales se ha contribuido a las propuestas en pro del adelanto de la provincia.

\section{El consumo de mora}

Para Marcelo (2014) se ha convertido en la fruta más demandada dentro de los hogares ecuatorianos especialmente por lo natural y saludable, en la que la Sociedad el consumo de frutos rojos ayuda a mantener el cerebro limpio. Nuevas investigaciones sugieren que una de las propiedades de estas frutas, como la fresa, es remover las toxinas que interfieren con las funciones cerebrales, son frutos bajos en
La mayor parte de cultivos de mora se encuentra en la Provincia de Tungurahua con 2.223 has (1.255 has como monocultivos y 968 como cultivos asociados), la cual corresponde al $42.36 \%$ del total nacional, el total de la producción es de 4.715 TM, que corresponde al $40.03 \%$ del total nacional y una cantidad vendida de 4.559 TM (39.66\% del total nacional).Sus zonas productoras son Tisaleo, Mocha, Ambato con los Cevallos, Pillaro, este cultivo se convierte en un sustento familiar (Ruiz, 2014).

Por ende el cantón Tisaleo es un referente provincial en la producción y comercialización de mora y fresa fresca de la más alta calidad de nuestro país por las tierras que contienen ceniza volcánica del Tungurahua, la misma que ha pasado su tiempo de transformación dotando a la tierra de buenas características para cultivos, dando como resultado un producto de buen sabor, color, tamaño además de su peso y precio justo

\section{Tecnificación del mercado agrícola}

En el Ecuador según el INEC (2015) en los cuatro últimos años se ha incrementado en un $10 \%$ el equipamiento de computadores portátiles mientras que los computadores de escritorio registran un $1,3 \%$, es importante resaltar que el $32.8 \%$ de los hogares a nivel nacional tienen acceso al internet; 10,3 puntos más que cuatro años atrás. En el área urbana el crecimiento es de 9,6 puntos, mientras que la rural de 8,9 puntos. Así también según el INEC (2015), el 32\% de los hogares tienen acceso al internet, de ellos el 21,3\% accede a través de algún medio inalámbrico, 1,2 puntos más que el 2012.

Como complemento a lo antes mencionado es necesario recalcar que el $65,6 \%$ de las personas que usa Internet lo hacen por lo menos una vez al día, seguidos de los que por lo menos lo utilizan una vez a la semana con el 30,4\%, lo que representa una buena probabilidad de obtener mayor cantidad de visitas utilizando este medio de

comunicación para ofertar productos. 
En la actualidad existen herramientas que permiten al agricultor y a su producto posicionarse en el mercado de una manera técnica y competitiva mejorando sus tiempos de producción, calidad y precio. La globalización ha permitido que el uso de la tecnología esté a disposición de una gran cantidad de grupos humanos de tal manera que la misma contribuye al impulso del comercio de productos y servicios (Mendoza, 2019). Dando a conocer los beneficios y bondades de los mismos a través de plataformas visuales y productos gráficos tangibles que establecen mayor reconocimiento y así posicionando a un producto en un mercado óptimo que capte al público objetivo acertado, fortaleciendo la producción frutícola, apoyando la soberanía alimentaria y contribuir con el cambio de la matriz productiva. Así también las técnicas de seguridad industrial, busca brindar mejores condiciones del ambiente laboral para salvaguardar la seguridad de los productores (Aagrosíntesis, 2016).

El objetivo es desarrollar una integración económica, productiva y social en dicho cantón el cual permita que las familias obtengan mayores ganancias con la venta de sus productos, pudiendo cubrir el mercado a nivel nacional, utilizando una adecuada campaña publicitaria y utilizando los recursos técnicos y tecnológicos de actualidad, generando un impacto social, económico y productivo con resultados amplios en cuanto a la producción y comercialización de mora y fresa.

\section{Teoría sobre la cual se sustentó la investigación}

En este contexto una vez analizada la situación actual de los agricultores de fresa y mora del cantón de Tisaleo, se determina que las carreras de Ingeniería en Ciencias de la Computación, Diseño Digital, Ingeniería
Industrial y Arquitectura pueden contribuir al desarrollo de este sector de la economía de Tisaleo, por lo cual está enfocado a optimizar los ingresos de los productores mediante el uso adecuado de las técnicas y las tecnologías para la comercialización de los productos así como la salud de los productores; en lo referente al aporte tecnológico una vez implantado servirá por mucho tiempo como constante mantenimiento de información. Finalmente, el diseño de la campaña publicitaria servirá por un largo plazo ya que los productos que se diseñen se los puede difundir varias veces.

\section{METODOLOGÍA}

Se planifica socializaciones con los agricultores de la fresa y mora del cantón Tisaleo, para informarles de todo el proceso para la realización del proyecto y llegar a acuerdos, debido a que el proyecto considera un diseño de una máquina artesanal, el cual se establece que el tiempo de vida será de 10 años en función de la depreciación de dicha máquina.

Para determinar el proceso se realiza la proyección de la maquina según los requerimientos que se identifican al estar insitu y como resultado de las reuniones con los agricultores.

\section{DIAGNÓSTICO}

Como primera actividad se planifica la realización de una matriz de verificación, de objetivos específicos cuyo propósito es dar a conocer la situación de los agricultores de la fresa y mora del cantón Tisaleo, es por ello, que se plantea preguntas con los indicadores que se establecen en la siguiente Tabla. 
Proyecto integral para agricultores de fresa y mora: Caso Tisaleo

Paulina Sánchez, Fernando Saá, Jorge Álvarez • VÍNCULOS-ESPE (2019) VOL. 4, No.3:30-38

Tabla 1

Matriz de Verificación

\begin{tabular}{|c|l|}
\hline$N^{\circ}$ & \multicolumn{1}{|c|}{ OBJETIVOS ESPECífICOS } \\
\hline 1 & $\begin{array}{l}\text { Socializar a los productores de Fresa y Mora del } \\
\text { cantón Tisaleo el uso de computación Básica e } \\
\text { Internet. }\end{array}$ \\
\hline 2 & $\begin{array}{l}\text { Desarrollar una herramienta de software para la } \\
\text { promoción de sus productos, el manejo y consulta de la } \\
\text { información del nuevo centro de acopio de productores } \\
\text { de Tisaleo. }\end{array}$ \\
\hline 3 & $\begin{array}{l}\text { Desarrollar una campaña publicitaria con apoyo de } \\
\text { la Tecnología para la difusión y comercialización de } \\
\text { fresa y mora que permita expandir el mercado de los } \\
\text { productos. }\end{array}$ \\
\hline 4 & $\begin{array}{l}\text { Verificar en las plantaciones el buen uso y manejo de } \\
\text { químicos (plaguicidas). }\end{array}$ \\
\hline 5 & $\begin{array}{l}\text { Evaluar el riesgo ergonómico por posturas forzadas, } \\
\text { movimientos repetitivos y manipulación manual de } \\
\text { cargas. }\end{array}$ \\
\hline 6 & $\begin{array}{l}\text { Diseñar y construir una máquina semiautomática para } \\
\text { la extracción de pulpa. }\end{array}$ \\
\hline
\end{tabular}

Dicho modelo, utiliza objetivos específicos como medio para que los agricultores de la fresa y mora del cantón Tisaleo conozca cuál es su situación con respecto a los seis criterios que lo componen y a partir de ese cotejo saber cuáles son sus puntos fuertes y también cuáles son aquellas áreas donde se hace preciso implantar acciones de mejora para una buena comercialización a futuro.

\section{RESULTADOS}

Una vez conocida la situación actual se plantea indicadores de resultados a alcanzar.

Tabla 2

Indicadores de resultados a alcanzar: cualitativos y cuantitativos

\begin{tabular}{|l|l|l|l|}
\hline Cronograma & $\begin{array}{l}\text { Indicadores } \\
\text { Verificables } \\
\text { Objetivamente }\end{array}$ & $\begin{array}{l}\text { Productos o } \\
\text { Resultados Alcanzados }\end{array}$ & $\begin{array}{l}\text { Nivel de } \\
\text { Cumplimiento } \\
\%\end{array}$ \\
\hline Meta 1 & & & $15 \%$ \\
\hline Actividad 1.1 & Acta & Firma del convenio & \\
\hline Actividad 1.2 & Definición & Temas definidos & \\
\hline Actividad 1.3 & Logística & Cronograma & \\
\hline Actividad 1.4 & Socializar & $\begin{array}{l}\text { Agricultores } \\
\text { capacitados }\end{array}$ & \\
\hline Actividad 1.5 & Diseñar & Planificación & \\
\hline Actividad 1.6 & Elaborar & Manual & \\
\hline Actividad 1.7 & Capacitación & Capacitación & \\
\hline Meta 2 & & & \\
\hline Actividad 2.1 & Recopilar & $\begin{array}{l}\text { Requerimientos del } \\
\text { sistema }\end{array}$ \\
\hline Actividad 2.2 & Diseñar & Esquema & \\
\hline Actividad 2.3 & Diseñar & Interfaz de usuario & \\
\hline Actividad 2.4 & Codificación & Código fuente & \\
\hline Actividad 2.5 & Pruebas & Retroalimentación & \\
\hline Actividad 2.6 & Instalación & Implementación & \\
\hline
\end{tabular}


La difusión del proyecto se mantendrá a través del tiempo con una duración de 3 a 5 años, por los cambios del contexto y requiriendo actualización de información.

Tabla 3

Indicadores de resultados a alcanzar: cualitativos y cuantitativos

\begin{tabular}{|c|c|c|c|}
\hline Cronograma & $\begin{array}{l}\text { Indicadores } \\
\text { Verificables } \\
\text { Objetivamente }\end{array}$ & $\begin{array}{l}\text { Productos o } \\
\text { Resultados } \\
\text { Alcanzados }\end{array}$ & $\begin{array}{l}\text { Nivel de } \\
\text { Cumplimiento } \\
\%\end{array}$ \\
\hline Meta 3 & & & 25 \\
\hline Actividad 3.1 & Proceso análisis & Tabla & \\
\hline Actividad 3.2 & $\begin{array}{l}\text { Proceso, lluvia de } \\
\text { ideas }\end{array}$ & Matriz & \\
\hline Actividad 3.3 & $\begin{array}{l}\text { Proceso, lluvia de } \\
\text { ideas }\end{array}$ & Listado & \\
\hline Actividad 3.4 & $\begin{array}{l}\text { Bocetaje, } \\
\text { digitalización y } \\
\text { pruebas. }\end{array}$ & $\begin{array}{l}\text { Impresos/pruebas de } \\
\text { color }\end{array}$ & \\
\hline Actividad 3.5 & $\begin{array}{l}\text { Bocetaje, } \\
\text { digitalización y } \\
\text { pruebas. }\end{array}$ & Impresos/ pruebas & \\
\hline Actividad 3.6 & $\begin{array}{l}\text { Desarrollo de } \\
\text { diseño }\end{array}$ & Digitales & \\
\hline Actividad 3.7 & Maquetación & Digitales e impresos & \\
\hline Actividad 3.8 & Puesta en escena & Impresos y digitales & \\
\hline Meta 4 & & & $10 \%$ \\
\hline Actividad 4.1 & $\begin{array}{l}\text { Creación del } \\
\text { check list }\end{array}$ & Check list & \\
\hline Actividad 4.2 & Temario & $\begin{array}{l}\text { Contenidos y temas } \\
\text { de capacitación }\end{array}$ & \\
\hline Actividad 4.3 & Logística & Cronograma & \\
\hline
\end{tabular}

\begin{tabular}{|c|c|c|c|}
\hline Actividad 4.4 & Capacitación & Capacitación & \\
\hline Meta 5 & & & $10 \%$ \\
\hline Actividad 5.1 & Procesos & $\begin{array}{l}\text { Diagrama de } \\
\text { procesos }\end{array}$ & \\
\hline Actividad 5.2 & Información & $\begin{array}{l}\text { Registro, fotografías } \\
\text { y videos }\end{array}$ & \\
\hline Actividad 5.3 & Software & Tabla de resultados & \\
\hline Actividad 5.4 & Medidas & $\begin{array}{l}\text { Información } \\
\text { comparativa con la } \\
\text { norma }\end{array}$ & \\
\hline Actividad 5.5 & Manual & Manual & \\
\hline
\end{tabular}

Tabla 4

Indicadores de resultados a alcanzar: cualitativos y cuantitativos

\begin{tabular}{|c|c|c|c|}
\hline Cronograma & $\begin{array}{l}\text { Indicadores } \\
\text { Verificables } \\
\text { Objetivamente }\end{array}$ & $\begin{array}{l}\text { Productos o } \\
\text { Resultados } \\
\text { Alcanzados }\end{array}$ & $\begin{array}{l}\text { Nivel de } \\
\text { Cumplimiento \% }\end{array}$ \\
\hline Meta 6 & & & $25 \%$ \\
\hline Actividad 6.1 & Identificar & $\begin{array}{l}\text { Detalle de } \\
\text { necesidades }\end{array}$ & \\
\hline Actividad 6.2 & Diseñar & $\begin{array}{l}\text { Diseño de } \\
\text { máquina artesanal }\end{array}$ & \\
\hline Actividad 6.3 & Seleccionar & $\begin{array}{l}\text { Datos técnicos de } \\
\text { materiales }\end{array}$ & \\
\hline Actividad 6.4 & Costo & Cotización & \\
\hline Actividad 6.5 & Construir & $\begin{array}{l}\text { Máquina } \\
\text { semiautomática } \\
\text { de extracción de } \\
\text { pulpa }\end{array}$ & \\
\hline TOTAL & & & $100 \%$ \\
\hline
\end{tabular}


Por tratarse de un proyecto cuyos resultados son en parte tangibles y en parte intangibles, se garantiza que, en el caso de la Capacitación los productores recibirán cursos según los requerimientos actuales y futuros en cuanto al uso de las tecnologías, cuyos contenidos les servirá para involucrarse en mundo actual y les permitirá acceder a capacitaciones futuras según el avance tecnológico. En lo referente al aporte tecnológico una vez implantado servirá por mucho tiempo a los con constante mantenimiento de información. Finalmente, el diseño de la campaña publicitaria servirá por un largo plazo ya que los productos que se diseñen se los puede difundir varias veces.

Así mismo, como resultado de las actividades realizadas por los grupos de interés, se tiene la materialización de la máquina despulpadora que presta servicios a la comunidad de manera intermitente, entre los socios de Panzaleo.

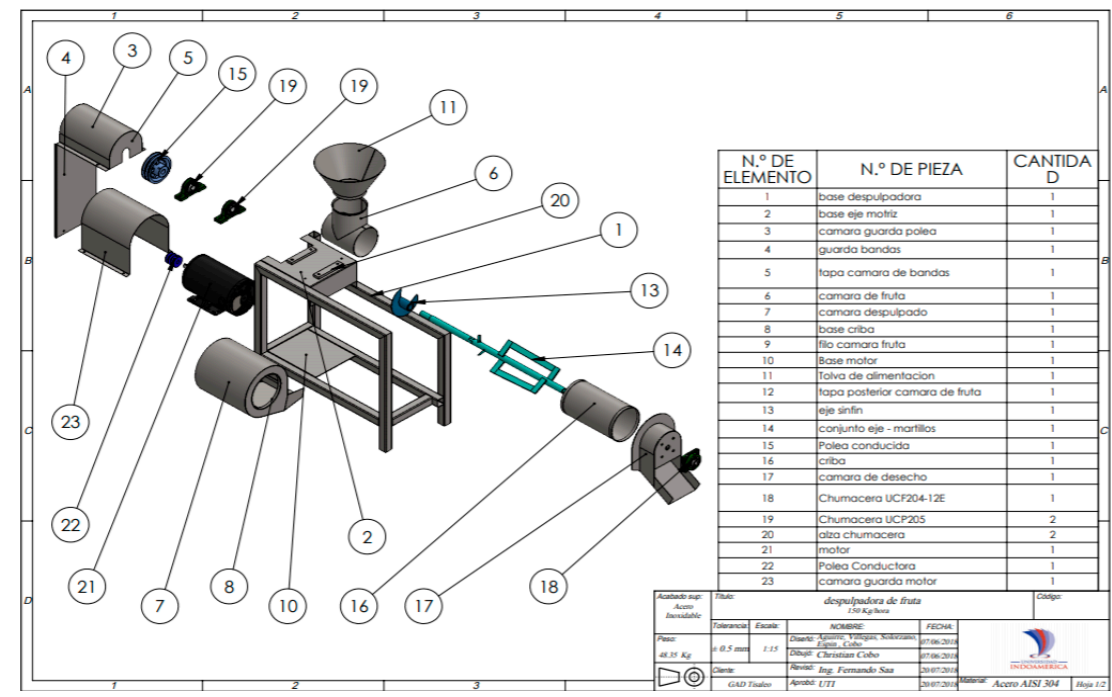

Fig. 1. Vista de explosión de la maquina despulpadora.

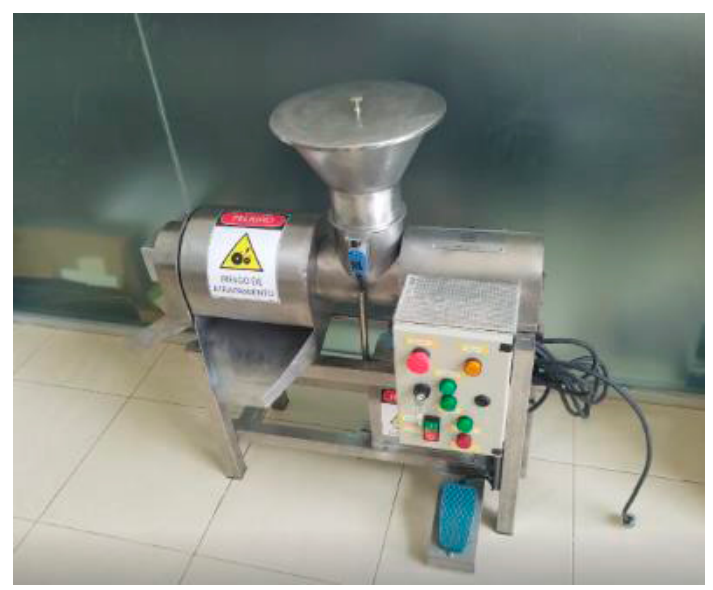

Fig. 2. Máquina despulpadora

Las especificaciones técnicas de la máquina despulpadora son las siguientes:

Tabla 1

Datos técnicos de la Máquina despulpadora.

\begin{tabular}{|l|l|}
\hline Medidas & \\
\hline Ancho & $441.14 \mathrm{~mm}$ \\
\hline Largo & $942.14 \mathrm{~mm}$ \\
\hline Alto & $923.39 \mathrm{~mm}$ \\
\hline Peso aproximado & $70 \mathrm{~kg}$ \\
\hline Características eléctricas & \\
\hline Voltaje & $110 / 220 \mathrm{~V}$ \\
\hline Amperaje & $15.40 / 7.70 \mathrm{~A}$ \\
\hline Frecuencia & $60 \mathrm{~Hz}$ \\
\hline Velocidad & $1700 \mathrm{rpm}$ \\
\hline
\end{tabular}


La Asociación San Miguel, del Centro Cantonal, está interesada en la máquina.

- Demanda actual 300 litros / semana.

- 300 litros / 5 días= 60 litros/día

- 60 I. / 8 horas diarias = 7,5 litros / hora

- La máquina debe erogar 12 litros / hora.

- 8 litros/hora, por $(1,5)$ factor de incremento demanda $=12$ litros/hora.

La optimización en dimensiones y simplicidad en el diseño, permitió reducir el gasto de horas hombre y costo por material en el orden del $36 \%$.

\section{CONCLUSIONES}

La demanda de producto en el mercado ha experimentado un continuo crecimiento durante los últimos años, con lo cual la principal estrategia de posicionamiento será: excelente calidad de servicio a precios muy competitivos. Para esto se ha logrado dotar de infraestructura técnica y tecnológica a través de las capacitaciones y el desarrollo tecnológico de la despulpadora.
Los estudios preliminares determinan que la situación en la que se encontró la Feria de fresa y Mora llevada a cabo en el Cantón Tisaleo hace 9 años, proyecta gran rentabilidad al estar situada en el centro del país; no ha sido difundida de manera correcta la misma que requería una actualización de identificador visual y un plan de campaña. Con esto se ha realizado un diseño corporativo en la marca y su posterior comercialización.

El desarrollo de una aplicación web permitirá dar a conocer al público en general la existencia de la producción de fresas y moras en el cantón Tisaleo, además automatizara procesos administrativos en el control del mercado de expendio que antes se los realizaba manualmente.

Al utilizar pesticidas químicos se produce desechos plásticos provenientes de los contenedores de estos, los cuales son quemados en su gran mayoría produciendo grandes consecuencias al medio ambiente; otros de los destinos de estos desechos son las quebradas o las acequias del sector produciendo contaminación.

\section{REFERENCIAS}

Aagrosíntesis. (30 de Mayo de 2016). Tecnificación para impulsar la productividad del campo. Obtenido de https://www.agrosintesis. com/tecnificacion-para-impulsar-la-productividad-del-campo/\# . XlxnLaAh3IU 
Ascolani, A. (2016). las categorías "proceso de tecnificación" y "proceso de civilización" contrastadas en el estudio de la sociedad agraria de un país periférico. . Argentina: Universidad Nacional de Rosario.

Hruska, A. (2013). Agricultura familiar y acceso a los mercados. Panamá: Fao.

INEC. (2015). Compendio Estadístico. Obtenido de http://www. ecuadorencifras.gob.ec/compendio-estadistico-2015/

Marcelo, B. A. (2014). La cadena de comercialización de la mora (Rubustglaucus benth) y la incidencia en el nivel de ingresos de los productores en la provincia de Tungurahua. Ecuador: UTA.

Mendoza, A. V. (15 de Marzo de 2019). La tecnificación agrícola.
Obtenido de https://www.eltelegrafo.com.ec/noticias/68/1/latecnificacion-agricola-2

ONUA. (2017). El Estado mundial de la agricultura y la alimentación. Roma: Organización de las Naciones Unidas para la Alimentación y la Agricultura.

Ruiz, M. M. (2014). Situación Actual y Perspectivas del Mercado de la Mora. Ecuador: Usaid.

U. T. Indoamérica. (2018). Universidad Indoamérica participó en Congreso de Vinculación con proyectos comunitarios que benefician a productores del cantón Tisaleo. Obtenido de http://www.uti.edu.ec/ universidad-indoamerica-participo-en-congreso-de-vinculacion-conproyectos-comunitarios-que-benefician-a-productores-del-cantontisaleo/

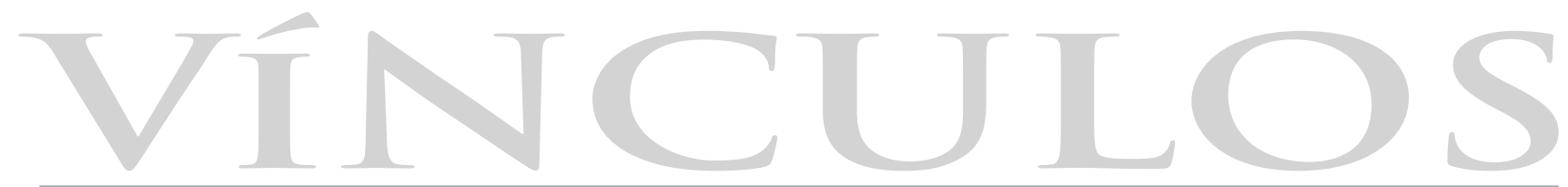

lat

UNIVERSIDAD DE LAS FUERZAS ARMADAS ESPE 